\title{
Tratamiento conservador de la osteoartrosis de cadera con toxina botulí́nica tipo A
}

\author{
Sofía Durán-Hernández ${ }^{*}$, Juan Francisco Soto-Rodríguez², Jorge Allen-Bobadilla ${ }^{3}$, \\ Nayar Durán-Hernández y Pamela García-Mosco ${ }^{1}$ \\ ${ }^{1}$ Servicio de Medicina Física y Rehabilitación; ${ }^{2}$ Servicio de Ortopedia y Traumatología, Hospital General Tacuba, ISSSTE, ${ }^{3}$ Práctica privada; Ciudad \\ de México, México
}

\section{Resumen}

Introducción: La osteoartritis de cadera es una enfermedad articular inflamatoria de bajo grado y etiología múltiple que tiene un manejo conservador limitado y poco explorado. La aplicación de toxina botulínica tipo A (BoNT-A) produce en el músculo aplicado una parálisis flácida temporal, delimitable y reversible, que aplicada de manera estratégica logra liberar estrés mecánico y dolor. Objetivo: Evaluar el efecto en la percepción de dolor, funcionalidad y rigidez y los cambios en la flexión, rotación interna y externa, antes y 90 días después de la aplicación de BoNT-A. Métodos: Estudio clínico, experimental, longitudinal, abierto, con un total de 35 pacientes y 45 caderas tratadas. Se aplicó BoNT-A de 500 U en músculos ilíaco, músculo aductorbrevis y longus. Resultados: Se utilizó la prueba de Wilcoxon comparando los puntajes de evaluación en los días 0 a 90 observando disminución significativa del dolor referido $(p<0.0001)$, rigidez $(p<0.002)$, mejoró la función percibida $(p<$ 0.001) y los arcos de movilidad: flexión, rotación interna y externa $(p<0.0001)$. Conclusiones: $E$ El tratamiento con BoNT-A otorga una opción conservadora y segura para el manejo de los síntomas y restricción física causada por la OA de cadera.

PALABRAS CLAVE: Osteoartritis. Toxina Botulínica. Dolor crónico.

\begin{abstract}
Introduction: Osteoarthritis $(O A)$ of the hip is a low-grade inflammatory disease of multiple etiology that has a limited conservative management and insufficiently explored. The application of botulinum toxin type $A$ (BoNT-A) produces in the applied muscle a temporary, delimitable and reversible flaccid paralysis, which applicated in a strategic way achives to reduce mechanical stress and pain. Objective: To evaluate the effect on pain perception, functionality and rigidity and changes in flexibility, internal and external rotation, before and 90 days after the application of BoNT-A. Methods: Clinical, experimental, longitudinal, open study, with a total of 35 patients and 45 hips treated. BoNT-A of $500 \mathrm{U}$ was applied in iliac, adductor brevis and longus muscles. Results: The Wilcoxon test was used to compare the evaluation scores on days 0-90, observing the decrease in referred pain $(p<0.0001)$, rigidity $(p<0.002)$, improved perceived function $(p<0.001)$ and mobility arcs: flexion, internal and external rotation $(p<0.0001)$. Conclusions: The treatment with BoNT-A provides a conservative and safe option for the management of symptoms and physical restraint caused by hip $O A$.
\end{abstract}

KEY WORDS: Osteoarthritis. Botulinum toxin. Chronic pain.

Correspondencia:

*Sofía Duran-Hernández

E-mail: dra.sofiaduranhernandez@gmail.com
Fecha de recepción: 11-03-2019

Fecha de aceptación: 19-03-2019

DOI: 10.24875/GMM.19005151
Gac Med Mex. 2019;155(Suppl 1):S56-S61

Disponible en PubMed www.gacetamedicademexico.com 


\section{Introducción}

La osteoartritis $(\mathrm{OA})$ es la artropatía más común en el mundo. Afecta a las articulaciones sinoviales y produce destrucción del cartílago articular, remodelación del hueso subcondral e inflamación de la cápsula sinovial y tejidos blandos periarticulares. Las articulaciones más afectadas son la rodilla, cadera e interfalángicas de las manos'.

A nivel mundial, es considerada la $13 .^{\text {a }}$ causa de años vividos con enfermedad ${ }^{2}$ y la $11 .^{\text {a }}$ causa de discapacidad $^{3}$. En EE.UU., la prevalencia de edad y llega al $33.6 \%$ en adultos mayores de 65 años ${ }^{4}$. En México la OA es la $8 .^{a}$ causa de consulta en medicina familiar en el IMSS 5 y tiene una prevalencia nacional estimada del $10.51 \%$, con una mayor prevalencia en mujeres $(11.7 \%)$ que en hombres $(8.71 \%)^{6}$.

El diagnóstico de la $\mathrm{OA}$ de cadera sigue los lineamientos de 1991 del Colegio Americano de Reumatología (ACR, por sus siglas en inglés) y se aborda mediante la historia clínica, examinación física y exámenes de laboratorio y radiográficos. En el algoritmo se toma en cuenta el dolor de cadera más dos criterios de los siguientes: 1) velocidad de sedimentación globular $<20 \mathrm{~mm} /$ hora, 2) osteofitos femorales o acetabulares y 3) estrechamiento del espacio articular superior, axial y/o medial ${ }^{7}$. La evaluación del grado de severidad de la OA se realiza mediante la escala radiológica de Kellgren y Lawrence propuesta en 1957, que considera cuatro grados de severidad con un aumento progresivo del compromiso de la articulación (Tabla 1).

La OA previamente era considerada una enfermedad degenerativa y exclusiva del cartílago articular, actualmente se asume como una condición crónica e inflamatoria de bajo grado que afecta a la articulación en su totalidad ${ }^{8}$. Existen tres factores principales que participan en este proceso: las características inherentes de las células especializadas que componen la articulación, el estrés mecánico al que se ven sometidas y el efecto del entorno sistémico en ellas. La inervación, además de participar como receptor de daño, es un importante regulador de la inflamación, pues modula la permeabilidad vascular. Esta afirmación se basa en la observación de pacientes con artritis reumatoide que sufrieron paresia o parálisis del miembro afectado, cuyos síntomas se veían aminorados o resueltos en articulaciones previamente afectadas. Esto dio lugar a la reproducción del fenómeno en modelos animales produciendo artritis inflamatoria y posterior denervación quirúrgica. En estos experimentos se encontró alterada la expresión de genes involucrados en la permeabilidad vascular y la migración celular transendotelial, logrando la reversión de la inflamación?

Las opciones terapéuticas prequirúrgicas para la OA de cadera han sido revisadas por el ACR en el 2012. Se recomienda la inclusión de los pacientes a un programa de ejercicio cardiovascular y pérdida de peso en caso necesario. En cuanto a las medidas farmacológicas, se recomienda que los pacientes usen analgésicos por razón necesaria y que estos sean seleccionados en un gradiente de menor a mayor incidencia de efectos colaterales: paracetamol, analgésicos no esteroideos (AINE), tramadol e inyección intraarticular de corticoesteroides. Es importante mencionar que a diferencia de otros tipos de OA, esta guía no recomienda el uso de condroitín sulfato y glucosamina, AINE tópicos o inyecciones intraarticulares de hialuronato ${ }^{10}$.

Dentro de los tratamientos quirúrgicos no protésicos se encuentran las tenotomías parciales o completas de aductores y músculos insertados en la espina ilíaca anterior, con lo que se logra disminuir la tensión muscular y el estrés mecánico entre el acetábulo y la cabeza femoral (cirugía de Voss). Dado que la toxina botulínica tipo A (BoNT-A) provoca una denervación química, delimitada y transitoria con la subsecuente parálisis flácida del músculo estriado inyectado, lo que se logra entonces es un efecto similar al de dicha técnica aplicándolo solo en los aductores y la porción ilíaca del psoas ilíaco ${ }^{11}$.

La toxina botulínica tiene 7 distintos serotipos (AG). El mecanismo de acción reside en su carácter de proteasa, que le permite cortar las proteínas de transporte sináptico que intervienen en la exocitosis de neurotransmisores. La BoNT-A actúa específicamente cortando la vesícula de transporte SNAP-25 (proteína asociada al sinaptosoma de $25 \mathrm{kDa}$ ), que produce la secreción de acetilcolina. Este efecto selectivo es la razón de su empleo terapéutico en enfermedades caracterizadas por la hiperfuncionalidad de las terminales colinérgicas ${ }^{12,13}$. Clásicamente este efecto ha sido catalogado como local, aunque recientes investigaciones muestran que la toxina puede migrar transaxonalmente desde la terminal sináptica de manera retrógrada. Se ha reportado el efecto inhibitorio de la secreción de glutamato y sustancia $P$ en la astroglía del asta posterior en la médula espinal que 
Tabla 1. Escala radiológica de Kellgren y Lawrence para OA de cadera

\begin{tabular}{ll}
\hline Grado de OA & Características \\
\hline Grado I & (OA dudosa) implica el estrechamiento en el espacio articular medial y osteofitos alrededor de la cabeza femoral \\
Grado II & $\begin{array}{l}\text { (OA moderada) estrechamiento franco del espacio articular, osteofitos bien definidos y esclerosis ligera en la superficie } \\
\text { articular }\end{array}$ \\
Grado III & $\begin{array}{l}\text { (OA moderada) marcado estrechamiento del espacio articular, osteofitos, esclerosis subcondral, formación de quistes y } \\
\text { deformidad de la cabeza femoral y acetábulo }\end{array}$ \\
Grado IV & $\begin{array}{l}\text { (OA severa) contacto directo de las superficies articulares, esclerosis subcondral, quistes, deformidad marcada de la } \\
\text { cabeza femoral y acetábulo y osteofitos grandes }\end{array}$
\end{tabular}

podría inhibir parcialmente la sobreexcitación en la transmisión nociceptiva ${ }^{13}$.

En este contexto, se propuso el abordaje con BoNT-A en una condición de dolor crónico asociado a un marcado estrés mecánico como la $O A$ de cadera. Previamente empleada en OA de rodilla y cadera en animales y humanos, la toxina ofrece una opción innovadora y segura ${ }^{14,15}$. El propósito de este estudio es evaluar si la BoNT-A tiene un efecto analgésico al disminuir el estrés mecánico entre la cabeza femoral y el acetábulo, así como en la regulación de neurotransmisores relacionados con el dolor, como la sustancia $p$ y el glutamato, y valorar si existe un efecto benéfico en el tratamiento de estos pacientes durante el tiempo de efecto de la toxina (3 meses).

\section{Métodos y pacientes}

Se realizó un estudio clínico, experimental y longitudinal, abierto. Con una muestra no probabilística, captando a los pacientes en la consulta de Medicina Física y Rehabilitación del Hospital General Tacuba (ISSSTE) en el periodo de diciembre de 2016 a marzo de 2017. Se incluyeron pacientes mayores de edad, de ambos sexos con diagnóstico de OA según criterios del ACR y con grados de severidad II a IV según la escala de Kellgren y Lawrence con cualquier tiempo de evolución. Se excluyó a los pacientes que: a) habían recibido una inyección intraarticular en los últimos 6 meses; b) tuvieron condiciones que pudieran interferir con la eficacia del tratamiento (p. ej., infección local, sistémica); c) condiciones médicas que contraindican el uso de terapia con BoNT-A (coagulopatías, embarazo, lactancia, alergia conocida al tratamiento), y d) enfermedades concomitantes que interfirieran con el correcto análisis de los resultados (enfermedades neuromusculares asociadas, diagnóstico de enfermedades reumatológicas).

\section{Aspectos éticos}

El presente estudio fue elaborado de acuerdo con los principios de la declaración de Helsinki. Fue sometido a evaluación en el Comité Hospitalario de Investigación (CHI) y el Comité Hospitalario de Ética en Investigación (CHEI) del Hospital General Tacuba (número de aprobación y registro: 035-2016). Posteriormente fue registrado y aprobado por el Comité nacional de investigación del ISSSTE (número de registro 498-2016). Todos los pacientes fueron informados de manera verbal y escrita acerca de los detalles del protocolo de investigación, sus dudas fueron resueltas a cabalidad y únicamente se incluyó a aquellos que firmaron el consentimiento informado. El tratamiento para su enfermedad no fue de ninguna manera limitado en caso de no desear participar en el estudio.

\section{Diseño del estudio}

Los pacientes fueron citados a valoración el día 0 y el 90. En cada una de las dos consultas se aplicó el cuestionario de Western Ontario and McMaster Universities Arthritis Index (WOMAC) para evaluación de dolor (5 preguntas), rigidez (2 preguntas) y función (17 preguntas), con sistema Likert de 0 a 4 en cada pregunta. A mayor puntuación, se asume un mayor nivel de compromiso sintomático y discapacidad. Así mismo se empleó la escala visual analógica (EVA) para la evaluación del dolor durante las 24 horas previas a la consulta. La valoración funcional de los pacientes fue realizada por goniometría en flexión, rotación interna y rotación externa de cadera por el mismo evaluador.

Siguiendo los esquemas terapéuticos propuestos por el ACR, se implementó el tratamiento convencional no farmacológico para OA de cadera consistente en 5 días de terapia con calor local por 10 minutos, 
así como enseñanza y ejecución posterior de ejercicios de estiramiento de miembros pélvicos y fortalecimiento de músculos de rodilla a tobillo, glúteo menor, bíceps femoral, glúteo mayor y medidas de higiene articular.

\section{Aplicación intramuscular de BoNT-A}

\section{Medicamento}

Se diluyó toxina abobotulínica tipo A (Dysport, IPSEN de $500 \mathrm{UI}$ ) en $1 \mathrm{ml}$ de solución salina estéril. Se aplicó en todos los pacientes un total de $350 \mathrm{UI}$ $(0.7 \mathrm{ml})$ en el vientre ilíaco del músculo ilíaco del iliopsoas y $350 \mathrm{UI}$ en los músculos adductoris brevis y adductoris longus (175 $\mathrm{Ul}$ o $0.35 \mathrm{ml}$ en cada uno) con aguja de $27 \mathrm{G}$, de pulgada y media de longitud.

\section{Aplicación del tratamiento}

Se realiza mediante referencia anatómica por un experto en aplicación del medicamento. Se coloca al paciente en posición decúbito supina con realización de técnicas de asepsia y antisepsia en zonas a infiltrar para el músculo ilíaco del iliopsoas: se realiza palpación de la espina ilíaca anterosuperior colocando la aguja a $2 \mathrm{~cm}$ de distancia hacia abdomen y dirigiendo aguja hacia el borde interno del hueso ilíaco penetrando de 2 a $3 \mathrm{~cm}$ tratando de topar con él e inyectando el medicamento. Músculos adductoris brevis: se localiza el tendón de origen que va del borde inferior de rama isquiopúbica a tuberosidad isquiática y se mide distalmente 7 a $8 \mathrm{~cm}$ (o cuatro dedos por debajo del pubis). Para el adductor longus se introduce la aguja de 1 a $3 \mathrm{~cm}$ de profundidad y para el adductor brevis de 3 a $5 \mathrm{~cm}$ de profundidad en dosis mencionadas.

\section{Análisis de datos}

Fue realizado de manera cegada por un estadista no relacionado con los pacientes o el diseño del estudio. Se empleó el paquete estadístico SPSS ${ }^{\circledR}$ versión 23 para MacOS (SPSS Inc., Chicago, IL, EE.UU.). Se realizaron estadísticos descriptivos para variables demográficas y escalas evaluadas. Se realizaron pruebas de normalidad en variables cuantitativas y al no tener un ajuste adecuado, se emplearon pruebas no paramétricas para su análisis. Las mediciones de las variables al día 0 y 90 fueron comparadas con la
Tabla 2. Datos demográficos de los pacientes. Se muestra la mediana y desviación estándar de las variables demográficas. EI primer apartado considera una $\mathrm{n}=35$ pacientes. La puntuación de la escala radiológica de Kellgren y Lawrence contempla un total de $\mathbf{4 5}$ caderas

\begin{tabular}{|c|c|}
\hline \multicolumn{2}{|c|}{ Datos demográficos de los pacientes } \\
\hline $\begin{array}{l}\text { Edad, años, media (DE) } \\
\text { Rango }\end{array}$ & $\begin{array}{c}n=35 \\
62.57(10.79) \\
42-84\end{array}$ \\
\hline Sexo (femenino/masculino), $\mathrm{n}$ & $31 / 4$ \\
\hline $\begin{array}{l}\text { IMC, kg/m², media (DE) } \\
\text { Rango }\end{array}$ & $\begin{array}{c}29.14(3.61) \\
21.43-35.7\end{array}$ \\
\hline Afección bilateral/unilateral, $\mathrm{n}$ & $10 / 25$ \\
\hline $\begin{array}{l}\text { Kellgren-Lawrence, n (\%) } \\
\text { Grado II } \\
\text { Grado III } \\
\text { Grado IV }\end{array}$ & $\begin{array}{c}n=45 \\
12(26.7) \\
18(40) \\
15(33.3)\end{array}$ \\
\hline
\end{tabular}

prueba de rangos con signo de Wilcoxon para muestras pareadas. Los resultados fueron considerados estadísticamente significativos cuando el valor de $p<0.05$.

\section{Resultados}

Se incluyó a un total de 39 pacientes, de los cuales 35 concluyeron el estudio. Diez pacientes con coxartrosis bilateral fueron incluidos, por lo que se considera un total de 45 caderas en las variables clínicas. Los pacientes se encontraron entre la $5 .^{a}$ y 9. ${ }^{a}$ década de la vida, con un porcentaje mayor de pacientes femeninos (88.57\%). El promedio de los pacientes se encontró en sobrepeso. Los porcentajes de los grados de severidad de las caderas incluidas fueron II (26.7\%), III (40\%) y IV (33.3\%) (Tabla 2).

Se encontró una disminución franca en las puntuaciones de la EVA, y en cada uno de los apartados y la puntuación total del cuestionario de WOMAC. Las variables de exploración física mostraron así mismo una franca mejora, con un aumento en la capacidad de flexión, rotación interna y rotación externa mediante la medición por goniometría. En todos los casos, se encontraron diferencias estadísticamente significativas (Tabla 3).

\section{Discusión}

La BoNT-A es un medicamento ampliamente utilizado para distonía y espasticidad muscular en niños y adultos, que logra mejorar la funcionalidad, 
Tabla 3. Valores basales y en el día 90 en las escalas de evaluación y exploración física $(n=45)$

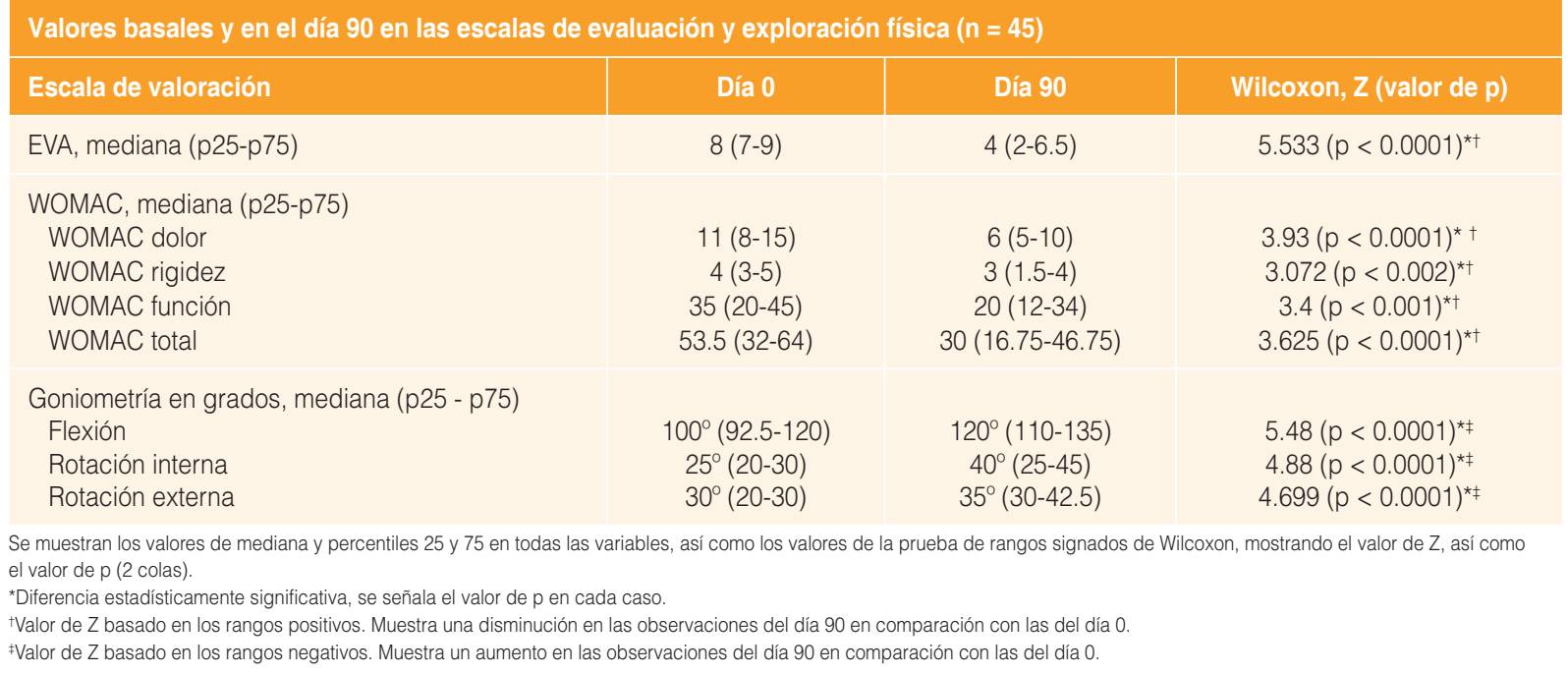

movilidad y disminución de dolor incrementando su calidad de vida ${ }^{16}$. Derivado a este uso se detectó su influencia en el control de dolor tipo neuropático en dichos pacientes y estudios a nivel básico que ponen en evidencia el control de sustancia $P$ y glutamato, relacionadas al sistema de liberación presináptico del SNAP-25 ${ }^{17}$.

Si bien en el presente estudio se logra un efecto de liberación de compresión articular relajando algunos de los músculos estabilizadores y cambiando de manera temporal el eje de carga, se debe de resaltar que los pacientes manifestaron un alivio del dolor, e incremento en los rangos de movimiento inmediatos posiblemente relacionado con el efecto analgésico referido previamente.

Marchiani, et al. publicaron en 2010 un estudio del manejo de coxartrosis con BoNT-A en el que se logró una disminución significativa del dolor referido y un aumento en la función aplicando el tratamiento en el músculo adductor longus y magnus de cadera un total de 400 unidades de BoNT-A. La muestra en dicho estudio fue de 39 pacientes y se administraron la EVA y el cuestionario Salud short form 36 (SF-36). Sin embargo, no se incluyó información acerca de artrometría como se realizó en el presente estudio ${ }^{14}$.

El presente estudio cuenta con las limitaciones de ser un diseño abierto y la falta de un grupo control por el hecho de haber sido un estudio piloto, el primero en México y el segundo reportado en el mundo. Sin embargo, abona evidencia al empleo de este tratamiento para el control a largo plazo del dolor crónico en la OA de cadera.
El manejo conservador de esta entidad nosológica a menudo falla por mala tolerancia a los ejercicios por el dolor, traslados largos para llegar al tratamiento y a su lugar de trabajo y domicilio, barreras arquitectónicas en lugar de trabajo, domicilio o incluso a nivel hospitalario, lo que provoca mayor estrés en el paciente, agotamiento y abandono del tratamiento.

\section{Conclusiones}

La aplicación de BoNT-A en músculo ilíaco y aductores brevis y longus disminuye el dolor y la rigidez referidas por el paciente e incrementa la funcionalidad percibida y encontrada a la exploración física. Es necesario continuar el estudio de las propiedades analgésicas de la toxina botulínica en estudios con mayores muestras, de diseño ciego y aleatorizado, y la integración de más variables clínicas, como el control imagenológico pre y postratamiento durante un tiempo prolongado.

\section{Conflicto de intereses}

Los autores declaran no tener conflictos de interés.

\section{Bibliografía}

1. National Clinical Guideline Centre. National Institute for Health and Clinical Excellence: Guidance. En: Osteoarthritis: Care and management in adults. Londres, Reino Unido: National Institute for Health and Care Excellence; 2014

2. GBD 2016 Disease and Injury Incidence and Prevalence Collaborators. Global, regional, and national incidence, prevalence, and years lived with disability for 328 diseases and injuries for 195 countries, 1990-2016: a systematic analysis for the Global Burden of Disease Study 2016. Lancet. 2017;390(10100):1211-59.

3. Cross M, Smith E, Hoy D, Nolte S, Ackerman I, Fransen M, et al. The global burden of hip and knee osteoarthritis: estimates from the global burden of disease 2010 study. Ann Rheum Dis. 2014;73:1323-30. 
4. Hootman JM, Helmick CG, Barbour KE, Theis KA, Boring MA. Updated projected prevalence of self-reported doctor-diagnosed arthritis and arthritis-attributable activity limitation among US adults, 2015-2040. Arthritis Rheumatol. 2016;68:1582-7.

5. Atención del paciente con osteoartritis de cadera y rodilla en el primer nivel. Secretaria de Salud Mexico [Internet]. México: gob.mx [fecha de última actualición: marzo de 2014]. Disponible en: https://cenetec-difusion.com/gpc-sns/?p=1287.

6. Pelaez-Ballestas I, Sanin LH, Moreno-Montoya J, Alvarez-Nemegyei J, Burgos-Vargas R, Garza-Elizondo M, et al. Epidemiology of the rheumatic diseases in Mexico. A study of 5 regions based on the COPCORD methodology. J Rheumatol. 2011:86:3-8.

7. Altman R, Alarcon G, Appelrouth D, Bloch D, Borenstein D, Brandt K et al. The American College of Rheumatology criteria for the classification and reporting of osteoarthritis of the hip. Arthritis Rheum. 1991; 34:505-14.

8. Robinson WH, Lepus CM, Wang Q, Raghu H, Mao R, Lindstrom TM et al. Low-grade inflammation as a key mediator of the pathogenesis of osteoarthritis. Nat Rev Rheumatol. 2016:12:580-92.

9. Ospelt $\mathrm{C}$, Frank-Bertoncelj M. Why location matters - site-specific factors in rheumatic diseases. Nat Rev Rheumatol. 2017;13:433-42.

10. Hochberg MC, Altman RD, April KT, Benkhalti M, Guyatt G, McGowan J, et al. American College of Rheumatology 2012 recommendations for the use of nonpharmacologic and pharmacologic therapies in osteoarthritis of the hand, hip, and knee. Arthritis Care Res (Hoboken). 2012;64:465-74.

11. Kollberg G, Lundholm G. The Voss operation in osteoarthritis of the hip. Acta Orthop Scand. 1965;36:82-8.

12. Filipovic B, Matak I, Bach-Rojecky L, Lackovic Z. Central action of peripherally applied botulinum toxin type $A$ on pain and dural protein extravasation in rat model of trigeminal neuropathy. PLoS One. 2012;7:e29803.

13. Marinelli S, Vacca V, Ricordy R, Uggenti C, Tata AM, Luvisetto S, et al. The analgesic effect on neuropathic pain of retrogradely transported botulinum neurotoxin A involves Schwann cells and astrocytes. PLoS One. 2012; 7:e47977

14. Marchini C, Acler M, Bolognari MA, Causero A, Volpe D, Regis D, et al. Efficacy of botulinum toxin type A treatment of functional impairment of degenerative hip joint: Preliminary results. J Rehabil Med;42:691-3.

15. Boon AJ Smith J, Dahm DL, Sorenson EJ, Larson DR, Fitz-Gibbon PD, et al. Efficacy of intra-articular botulinum toxin type A in painful knee osteoarthritis: a pilot study. PM \& R: the journal of injury, function, and rehabilitation. 2010;2:268-76.

16. Cubillos O, Maldonado A, Moyano Vera Á, San Martín E. Toxina botulínica y su importancia en el campo de la rehabilitación. Rev Hosp Clín Univ Chile 2010; 21: 319 - 25.

17. Cuevas-Trisan R, Motta-Valencia KJ. Botulinum toxins for pain management: A comprehensive review. J Rehabil Med. 2013;1:51-64. 